\title{
Quantum Particle Swarm Optimization Applied to Distinct Remuneration Approaches in Demand Response Programs
}

\author{
Fábio Pereira, João Soares, Pedro Faria, Zita Vale \\ GECAD - Knowledge Engineering and Decision Support Research Center \\ IPP - Polytechnic Institute of Porto \\ Porto, Portugal \\ faper@isep.ipp.pt, joaps@isep.ipp.pt, pnfar@isep.ipp.pt, zav@isep.ipp.pt
}

\begin{abstract}
The development of demand response programs has been allowing to improve power system performance in several ways, both in terms of the management of electricity markets, as well as regarding benefits in its operation. In order to model the remuneration for the participation of consumers in the scheduling of resources, this paper proposes a methodology based on the use of four incentive-based tariffs for the remuneration of demand response participation. It considers steps, quadratic, constant and linear remuneration. The optimization model enables Virtual Power Players to minimize operation costs, considering different critical situations of management and operation. The optimization problem has been solved by Quantum Particle Swarm Optimization. The case study concerns 168 consumers, classified into 5 consumer types, 118 distributed generation resources and 4 external suppliers.
\end{abstract}

Index Terms -Demand response programs, quantum particle swarm optimization, remuneration tariffs, virtual power player.

\section{NOMENCLATURE}

c Consumer index

$C_{\text {Const }} \quad$ Const program cost [m.u./kW]

$C_{D G} \quad$ Cost of distributed generation [m.u./kW]

$C_{L}^{b} \quad L$ program cost linear coefficient [m.u./kW]

$C_{L}^{c} \quad L$ program cost constant coefficient $[$ m.u./kW]

$C_{Q}^{a} \quad Q$ program cost quadratic coefficient [m.u./kW]

$C_{Q}^{b} \quad Q$ program cost linear coefficient [m.u./kW]

$C_{N S P} \quad$ Non-supplied Power cost [m.u./kW]

$C_{S} \quad S$ program cost component [m.u./kW]

The present work was done and funded in the scope of the following projects: EUREKA - ITEA2 Project SEAS with project number 12004; ELECON Project, REA grant agreement No 318912 (FP7 PIRSES-GA2012-318912); H2020 DREAM-GO Project (Marie Sklodowska-Curie grant agreement No 641794); and UID/EEA/00760/2013 funded by FEDER Funds through COMPETE program and by National Funds through FCT.

$\begin{array}{ll}C_{\text {Supplier }} & \text { Supplier power cost [m.u./kW] } \\ g & \text { Distributed generation index } \\ i ; i i ; i i i & \text { Each elementary step } \\ N C & \text { Total number of consumers } c \\ N G & \text { Total number of distributed generation } g \\ N S p & \text { Total number of suppliers } s p \\ P_{C o n s t} & \text { Const program reduction }[\mathrm{kW}] \\ P_{D G} & \text { Power schedule in distributed generation }[\mathrm{kW} \\ P_{L} & \text { L program reduction [kW] } \\ P_{\text {Load }} & \text { Power scheduled for the consumption }[\mathrm{kW}] \\ P_{N S P} & \text { Non-supplied Power [kW] } \\ P_{Q} & Q \text { program reduction [kW] } \\ P_{S} & S \text { program reduction }[\mathrm{kW}] \\ P_{\text {Supplier }} & \text { Supplier power [kW] } \\ S p & \text { Electricity Supplier index }\end{array}$

\section{INTRODUCTION}

Demand Response (DR) programs can bring several benefits, representing a way to cooperate in order to ensure the best operation of an electric power system. This concept is widely exploited in electricity markets, allowing to prevent possible critical situations, such as periods with high consumption [1]. On the other hand, the use of DR programs 
is able to enable the reduction of operation costs in certain operational scenarios of an electric network [2].

Currently, the strong presence of Distributed Generation (DG), particularly in distribution networks, allows to change the characteristics of management and operation of the electric power system [3]. The DG concerns a decentralization of power generation through small units that are connected and distributed in the network. Among the several benefits that such technologies bring, stand out the environmental benefits, because these are largely based in renewable sources. Moreover, they can allow greater reliability at the operational level, in cases of high energy demand or in situations where external suppliers, due to connection or generation problems, cannot meet consumption. However, DG based on renewable sources can present issues regarding continuity of supply [4].

In this way, the two concepts of DR programs and DG may be associated and, thus, allowing to provide a better service [5]. To this end, it is required an aggregator entity, designated by Virtual Power Player (VPP), to consider and make the management of the small size resources, both for consumption or generation. The VPP is able to aggregate small size consumers and generators, so that these can take an active role in the scheduling of resources [6].

In what concerns the aggregation of small size consumers, the VPP in addition to manage, should be able to develop models that attract consumers to participate in DR programs in order to reduce their consumption. DR programs can be pricebased or incentive-based [7]. In the first case, the consumers participate based on price signals, and consequently, change their consumption according to the current price of electricity. In the second case, the consumers receive additional incentives to participate in DR programs.

The remuneration of consumers should be appropriate to the characteristics that these present. In this way, it becomes important to study and develop models whose the available incentives are suitable for each type of consumer, which can be managed by VPP. Several studies have distinct models approaches to remunerate consumers, such as logarithmic or exponential models, as well as compensation based in elasticity models. These distinct approaches do not distinguish the several consumers, being applied the same remuneration model for all types of consumers [8]-[10].

The present paper shows a methodology that has been developed in order to a VPP can address a remuneration of according to each kind of consumers. The VPP is able to consider the use of DR programs in situations where can be most economically advantageous or critical situations network operation, such as power shortages or lines and other components outages, and situations of unpredictability of the DG resources. Among the several remuneration approaches of consumers, the VPP can use four different types, specifically, steps, quadratic, constant and linear. These distinct approaches aim to adjust the remuneration of each consumer and satisfy the classification that belong to incentive-based and to price-based.

The minimization of operation costs is obtained using Quantum Particle Swarm Optimization (QPSO). The implementation of QPSO allows to solve complex optimization problems, achieve fast and reasonably solutions [11], namely, in applications such as economic dispatch, unit commitment and optimal power flow problems [12]-[14].

This paper has as contributions an implementation of QPSO algorithm to minimize operation costs, considering the integration of several resources, such as DR, DG and external suppliers. The use of DR is characterized by the presence of four types of remuneration approaches, allowing the VPP associate an approach best suited to each consumer type.

After this introductory section, Section II details the main contributions of the paper and explains the proposed methodology. Then, Section III shows the mathematical formulation of the optimization problem, which includes the details on the QPSO approach. Section IV illustrates the case study and Section V includes the obtained results. Finally, Section VI presents the main conclusions of the work.

\section{PROPOSED DR PROGRAMS DEFINITION METHODOLOGY}

In this section, it is presented the explanation of the proposed methodology that has been developed to assist the VPP in decision making in the implementation of distinct approaches of DR programs.

The methodology proposed in the present paper aims to support Virtual Power Player (VPP) decision namely to minimize operation costs. The resource optimization developed by the VPP can consider using different approaches to DR programs, as well as DG and external supplier's resources to ensure the balance of the electric power system. VPP can schedule consumer participation both to ensure the reliability in critical situations of management and operation of electric power systems, such as, price increase of energy supplied, problems of connection between consumers and external suppliers and unavailability of wind.

In scheduling resources, the VPP is able to take into account the reduction of consumption and minimize operation costs. To this end, several types of consumer can participate in the consumption reduction. As each type of consumer has distinct characteristics, it is essential that the VPP provides different models to adequately remunerate the respective consumers. This way, the proposed methodology takes into account this aspect, differing from the other underlying literature [8]-[10].

The diagram presented in Fig. 1 illustrates the proposed methodology in which several scenarios are defined.

According to Fig. 1, the VPP considers several inputs to develop this methodology. Thus, the VPP takes into account the producers and consumers type, initial consumption forecast, capacity and forecast of each generation source and the limits considered in the DR resources.

Modelling of consumers includes four different types of remuneration, designated by steps, quadratic, constant and linear. This way, the VPP is able to discuss on the most appropriate remuneration in each context, for each type of consumer, through the implementation of DR programs that best suits their features. 


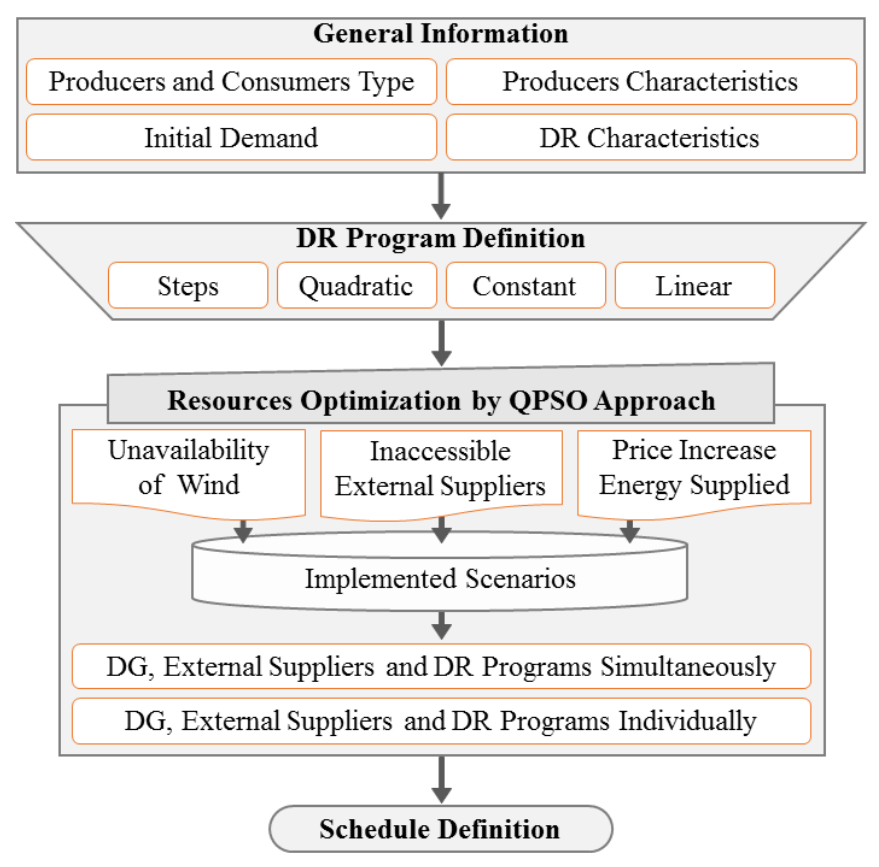

Figure 1. Diagram of the proposed methodology.

The VPP is able to perform an optimization of resources, based on Quantum Particle Swarm Optimization (QPSO). Subsection III-B the details this optimization approach. It is considered for optimal scheduling the DG, external suppliers and DR programs resources. Among the three scenarios developed for the determination of minimum operation costs, include operation critical situations, such as, price increase of energy supplied, problems of connection between consumers and producers (external suppliers) and consider unpredictability of DG resources, particularly, wind unavailable. In each one of scenarios described are applied in two distinct ways the DR programs, i.e., it is considered the implementation of DR programs individually, where can be associated any one of the consumers, or simultaneously, which each are associated the distinct consumers, in accordance with their characteristics.

The innovative aspects of the proposed methodology and contributions concerns the following aspects:

- Resources (DR, DG, and suppliers) use optimization performed from the VPP's standpoint aiming at the minimization of its operation costs;

- Modelling of four types of DR programs (linear, steps, quadratic and constant), for the remuneration of consumers;

- Definition of the most advantageous modelling approach in each context of consumption reduction need and for each consumer type;

- Optimal energy resource scheduling considering innovative constraints aiming the VPP to characterize the roles to be associated to each DR program and to a set of DR programs in which a certain consumer is participating, as well as integration of DG;

- Use of QPSO in optimization problems concerning DR programs definition.

\section{RESOURCE MANAGEMENT OPTIMIZATION}

This section presents the mathematical formulation in subsection III-A, on the problem of resource scheduling optimization, considering the several constraints that the VPP should manage. In subsection III-B, it is shown in detail the implementation of QPSO optimization technique.

\section{A. Mathematical Formulation}

The present subsection addresses the formulation of the optimization problem concerning the minimization operation costs, considering the usage costs of the DG, suppliers and DR programs resources. Using this optimization, the VPP is able to apply distinct DR programs approaches for adequate remuneration by each consumer. Simultaneously, the VPP is able to make economic decisions, scheduling the generation resources and implementation of the DR programs to satisfy possible critical situations.

The objective function of the VPP resource scheduling problem is given by equation (1), where the minimization operation costs (OC) includes the generation (DG and supplier) and DR programs costs related to the contribution power of each of them.

\section{Minimize $O C=$}

$$
\begin{aligned}
& {\left[\sum_{s p=1}^{N S p}\left(P_{\text {Supplier }(s p)} \times C_{\text {Supplier }(s p)}\right)+\sum_{g=1}^{N G}\left(P_{D G(g)} \times C_{D G(g)}\right)\right]} \\
& +\sum_{c=1}^{N C}\left[\begin{array}{l}
\left(P_{S(c)}^{i} \times C_{S(c)}^{i}+P_{S(c)}^{i i} \times C_{S(c)}^{i i}+P_{S(c)}^{i i i} \times C_{S(c)}^{i i i}\right) \\
+\left(P_{Q(c)}^{2} \times C_{Q(c)}^{a}+P_{Q(c)} \times C_{Q(c)}^{b}\right) \\
+\left(P_{\text {Const }(c)} \times C_{C o n s t(c)}\right) \\
+\left(P_{L(c)} \times C_{L(c)}^{b}+C_{L(c)}^{c}\right) \\
+\left(P_{N S P(c)} \times C_{N S P(c)}\right)
\end{array}\right]
\end{aligned}
$$

Equation (1) allows to demonstrate the characteristics of each of DR programs implemented. The DR steps program allows establish a contract with consumer, in which the VPP provides three prices $(i, i i$, iii), depending on amount of consumption reduction. In this way, the consumer that participates in this program is subject to receive remuneration, where each price level corresponds to a certain amount of power that can be reduced. The DR quadratic program is characterized by a quadratic trend in the remuneration to the consumer, including quadratic $(a)$ and linear $(b)$ coefficient. Lastly, the DR constant and linear programs have similar characteristics, however, the DR linear program envisages a fixed cost, representing a constant coefficient $(c)$.

The identification of DR programs is, therefore, based on the following acronyms: " $S$ - DR steps program"; " $Q$ - DR quadratic program"; "Const - DR constant program"; " $L$ - DR linear program".

The first constraint of the optimization problem to be considered by VPP, is the balance equation, identified in 
equation (2). This equation aims to ensure the system balance, considering the amount of load demand $\left(P_{\text {Load }}\right)$ to be met, as well as includes the DG, the external suppliers and the DR programs resources.

$$
\begin{aligned}
& \sum_{c=1}^{N C} P_{\text {Load }}=\sum_{s p=1}^{N S p} P_{\text {Supplier }(s p)}+\sum_{g=1}^{N G} P_{D G(g)} \\
& +\sum_{c=1}^{N C}\left[\begin{array}{l}
P_{S(c)}^{i}+P_{S(c)}^{i i}+P_{S(c)}^{i i i}+P_{\text {Const }(c)} \\
+P_{Q(c)}+P_{L(c)}+P_{N S P(c)}
\end{array}\right]
\end{aligned}
$$

The full formulation of the problem includes limitations that are imposed to power and price of each resource (producers and consumers). The model also contemplates a constraint that establishes the maximum consumption reduction by each consumer in what concerns the participation in each DR program approach. In addition, for each DR program is imposed a maximum limit of participation, both individually and as the sum of the participation of all DR programs are instituted a maximum reduction of consumption, considering the participation of each consumer.

\section{B. Quantum PSO}

Quantum PSO is a population-based stochastic method with different characteristics of those found in PSO [15]. The introduced exponential distribution of positions makes QPSO global convergent. The traditional PSO relies on the convergence to the global best (best solution of the swarm) particle, independent of the position of other particles. On the other hand, in the QPSO with mean best position, each particle cannot converge to global best position without considering its colleagues [15]-[16].

Fig. 2 illustrates how differently the lagging phenomena of QPSO and PSO particles are. In Fig. 2, the big blue circle represents the global best position while the little circles represent the other particles, and the little circles with vertical lines represents the lagged particles. The arrows around the little circles represent the possible directions of the particles; the big arrowhead points to the direction in which the particle moves with high probability.
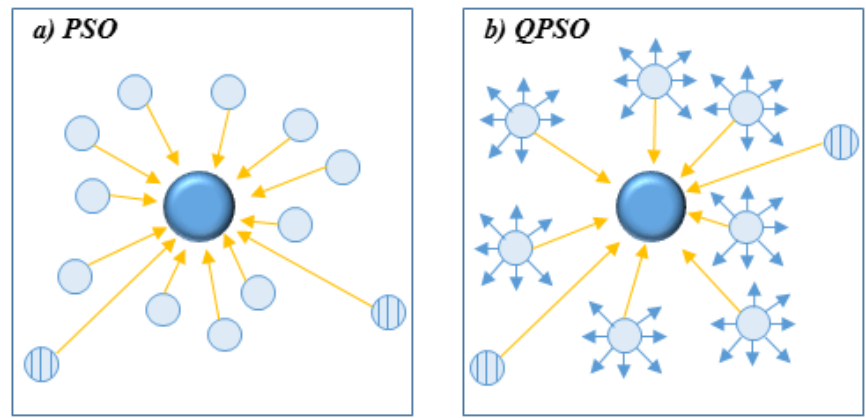

Figure 2. Lagged particles and waiting phenomena among particles in PSO and QPSO, based on [16].

In the PSO each particle flies towards the global best position without waiting for other particles. Each particle converges to the global best position independently without waiting for other particles. The influence of the lagged particles on the other particles is very little since the only connection is the global best position. In QPSO the lagged particles have greater influence on the other particles through mean best position. In the QPSO method the lagged particles are not abandoned by the swarm. The lagged particles affect the mean best position and therefore the lagged particles are shifted towards the rest of the swarm. The mean best position is intrinsically related with the movement equation of QPSO (4). The particles' distribution affects the convergence rate; however, QPSO can provide stronger global search ability than traditional PSO [15].

Equation (3) presents the local focus equation which is a random point located within the hyperplane constructed between $P_{i, n}$ and $G_{n}$ in the search space. The local focus is then used in equation (4). The contraction-expansion (CE) coefficient $\alpha$, which is vital to the dynamical behavior of an individual particle and the convergence of the algorithm. The algorithm decides to add or subtract the second term of equation (9) using a probability of $50 \%$.

$$
\begin{gathered}
p_{i, n+1}^{j}=\varphi_{i, n}^{j} P_{i, n}^{j}+\left(1-\varphi_{i, n}^{j}\right) G_{n}^{j} \\
X_{i, n+1}^{j}=p_{i, n}^{j} \pm \alpha\left|X_{i, n}^{j}-C_{n}^{j}\right| \ln \left(\frac{1}{u_{i, n+1}^{j}}\right)
\end{gathered}
$$

where:

$C_{n}^{j} \quad$ Mean best position which can be defined by the average of the personal best positions of all particles

$G_{n}^{j} \quad$ The global best of particle at the $n$th iteration

$P_{i, n}^{j} \quad$ The $j$ th component of the personal best of particle $i$ at the $n$th iteration

$p_{i, n}^{j} \quad$ The $j$ th component of the local focus $p_{i, n}$ of particle $i$ at the $n$th iteration

$u_{i, n+1}^{j} \quad$ Random uniformly distributed sequence [0,1]

$X_{i, n}^{j} \quad$ Position of the particle $i$ th at the $n$th iteration

$\varphi_{i, n}^{j} \quad$ Random uniformly distributed sequence [0,1]

\section{CASE STUdY}

The present section identifies in detail the case study that allows the VPP to apply the proposed methodology. This case study is based in [17], and it is characterized by being constituted by several power generation technologies and different types of consumers to be scheduled optimally.

The VPP of the demand side must take into account five types of consumers aggregates, i.e., the Domestic (DM), the Small Commerce (SC), the Medium Commerce (MC), the Large Commerce (LC), and the Industrial (I).

Among the 168 consumers present in the case study, the total capacity consumption is $12112 \mathrm{~kW}$, but in this case study was selected one period whose their total consumption was of $7326.58 \mathrm{~kW}$. The Fig. 2 shows the consumption and consumers by type. 


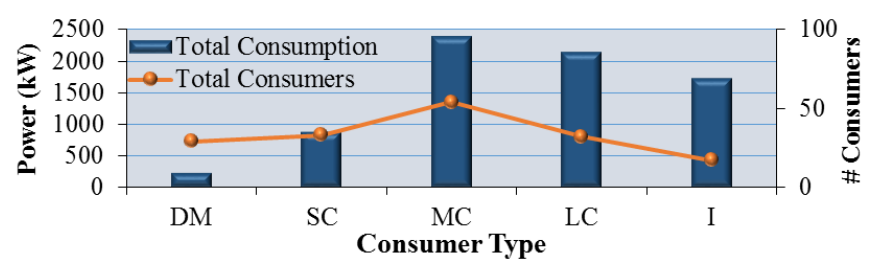

Figure 2. Total consumption and consumers by each type.

As explained in Section II and III, the several consumers in the present case study can be associated with distinct approaches of DR programs to suppress the several critical situations that may occur in the operation of a network. In this case study it is considered that there are three scenarios, each scenario corresponds, respectively: "Scenario A" - price increase of energy supplied from external suppliers; "Scenario B" - no supply from the upstream network, i.e. energy provided by external suppliers (simulation of connection network problems between consumers and external suppliers); "Scenario C" - unavailability of wind.

Each of these scenarios, it is also considered the association of DR programs in two different ways, individually or simultaneously. For the first case, each DR program can be applied the all consumers, and for the second case, each one of DR program approach is combined with specific consumers, i.e., only is available for each type of consumer two distinct approaches of DR programs, namely, Domestic (DM) and Small Commerce (SC) can be associated with the DR constant and linear programs, the Medium Commerce (MC) can be related to DR linear and steps programs, the Large Commerce (LC) and Industrial (I) can be combined with DR steps and quadratic programs.

Fig. 3 shows the limit of consumption reduction that each type of DR program allowed by each type of consumer.

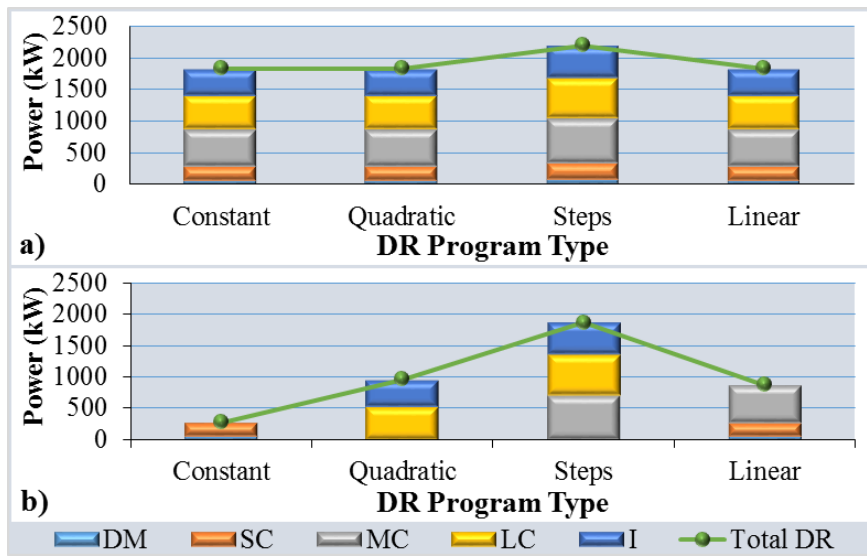

Figure 3. Maximum consumption reduction by DR program.

In a) it can be seen the maximum limit that each DR program can achieve when used individually. In b) it is shown the maximum limit that each DR program can have when DR programs are available simultaneous.

As can be seen in Fig. 3, each type of consumer can have at most two DR programs when implemented simultaneously. Note that in the simultaneous case the maximum consumption reduction by each program can be $40 \%$ of the total reduction.
Table I identifies the prices applied to each DR program in accordance with the type of consumer associated [5].

TABLE I. PRICES By CONSUMER AND DR PROGRAM TyPeS.

\begin{tabular}{|c|c|c|c|c|c|c|}
\hline \multicolumn{2}{|c|}{$\begin{array}{c}\text { Prices } \\
(m . u . / k W h)\end{array}$} & DM & SC & MC & LC & I \\
\hline \multicolumn{2}{|c|}{ Constant } & 0.20 & 0.16 & 0.19 & 0.18 & 0.14 \\
\hline \multirow{2}{*}{ Linear } & $b$ & 0.1980 & 0.1584 & 0.1881 & 0.1782 & 0.1386 \\
\hline & $c$ & 0.0020 & 0.0016 & 0.0019 & 0.0018 & 0.0014 \\
\hline \multirow{2}{*}{ Quadratic } & $a$ & 0.000020 & 0.000016 & 0.000019 & 0.000018 & 0.000014 \\
\hline & $b$ & 0.20 & 0.16 & 0.19 & 0.18 & 0.14 \\
\hline \multirow{3}{*}{ Steps } & $i$ & 0.16 & 0.15 & 0.18 & 0.17 & 0.17 \\
\hline & $i i$ & 0.20 & 0.19 & 0.20 & 0.24 & 0.26 \\
\hline & iii & 0.24 & 0.22 & 0.26 & 0.26 & 0.28 \\
\hline
\end{tabular}

In what concerns the generation data of the several DG technologies, Table II shows the features and prices considered by the VPP.

TABLE II. Generation CHARACTERISTICS AND PRICES.

\begin{tabular}{c|c|c|c|c|c}
\hline \hline $\begin{array}{c}\text { Type of } \\
\text { generator }\end{array}$ & $\begin{array}{c}\# \\
\text { Units }\end{array}$ & $\begin{array}{c}\text { Total } \\
\text { capacity } \\
(\boldsymbol{k W})\end{array}$ & $\begin{array}{c}\text { Forecast } \\
(\boldsymbol{k W})\end{array}$ & $\begin{array}{c}\text { Min price } \\
(\boldsymbol{m} . \boldsymbol{u} . / \boldsymbol{k} \boldsymbol{h})\end{array}$ & $\begin{array}{c}\text { Max price } \\
(\boldsymbol{m} . \boldsymbol{u} . / \mathbf{k} \boldsymbol{h})\end{array}$ \\
\hline Photovoltaic & 82 & 3540.00 & 223.49 & - & 0.2 \\
\hline Cogeneration & 4 & 4000.00 & 4000.00 & 0.1 & 0.12 \\
\hline Small Hydro & 1 & 1320.00 & 143.51 & - & 0.13 \\
\hline Wind & 30 & 2570.00 & 905.34 & - & 0.12 \\
\hline Biomass & 1 & 520.00 & 520.00 & - & 0.15 \\
\hline $\begin{array}{c}\text { External } \\
\text { Supplier 1 }\end{array}$ & 1 & 1500.00 & 1500.00 & - & 0.11 \\
\hline $\begin{array}{c}\text { External } \\
\text { Supplier 2 }\end{array}$ & 1 & 1000.00 & 1000.00 & - & 0.13 \\
\hline $\begin{array}{c}\text { External } \\
\text { Supplier 3 }\end{array}$ & 1 & 2000.00 & 2000.00 & - & 0.17 \\
\hline $\begin{array}{c}\text { External } \\
\text { Supplier 4 }\end{array}$ & 1 & 2800.00 & 2800.00 & - & 0.21 \\
\hline \begin{tabular}{c} 
Total \\
\hline \hline
\end{tabular} & 122 & 19250.00 & 13102.34 & - & - \\
\hline
\end{tabular}

Regarding photovoltaic and wind technologies, the VPP must consider the power forecast. The forecast considered in this case study for the photovoltaic technology is $223.49 \mathrm{~kW}$, while the forecast for wind is $905.34 \mathrm{~kW}$, as can be seen in Table II. Thus, the VPP scheduling must always consider the available energy for photovoltaic and wind power generation, while for others technologies of generation, the VPP can schedule according to the envisaged needs.

The focus of this study is to allow the VPP to consider several scenarios that allow to characterize technical constraints. Among the scenarios considered we stand out the impossibility of resorting to external suppliers by connection problems between loads and suppliers, as well as unavailability of wind that was previously expected. To address these critical situations, the VPP is able to make use of the remaining generation resources available and DR resources, using the previously described DR programs.

In what concerns the QPSO was implemented in MATLAB, and the modelling used in the present case study includes 1130 variables. Table III presents the parameters 
considered in QPSO approach, among them, the number of particles is 20 and maximum number of iterations was set to 10000. Eventually, the method might stop earlier if the fitness evolution is not changing over the last 4000 iterations more than $1^{-9}$. It is also contemplated the alpha value, which is important to control method's convergence. This parameter decreases linearly between 1.0 and 0.3 during swarm iterations.

The initial solution of the method is generated using an order of merit scheme, in which the resources with lower costs are scheduled first.

TABLE III. PARAMETER OF QPSO APPROACH.

\begin{tabular}{c|c|}
\hline \hline Parameters & Value \\
\hline \# Individuals & 20 \\
\hline Initial Solution & Random \\
\hline \#Iterations & 10000 \\
\hline Stopping Criteria & $\begin{array}{c}\text { Max. 10000 iterations Min. 5000 iterations and } \\
\text { fitness threshold }\left(1^{-9}\right) \text { during last 4000 iterations }\end{array}$ \\
\hline Maximum Positions & Equal to the upper bound of variables \\
\hline Minimum Positions & Equal to the lower bound of variables \\
\hline Alpha $(\alpha)$ & 1.0 decreasing to 0.3 \\
\hline \hline
\end{tabular}

\section{RESULTS}

The present section shows the results obtained with the proposed methodology used in the case study of Section IV. In subsection $\mathrm{V}$-A, we present the results for each of the three scenarios of operation critical situations, when DR programs are implemented simultaneously, and in subsection V-B is shown the results when DR programs are associated individually. Finally, in subsection V-C is presented the results analysis concerning the comparison between two distinct ways of implementation of DR programs.

\section{A. Implementation of DR Programs Simultaneously}

The results of implementation of DR programs resources in simultaneous are presented in detail during this subsection. In Fig. 4 it can be seen the convergence performance of the QPSO algorithm, considering 1000 random runs whose inputs from of scenario of wind unavailable.

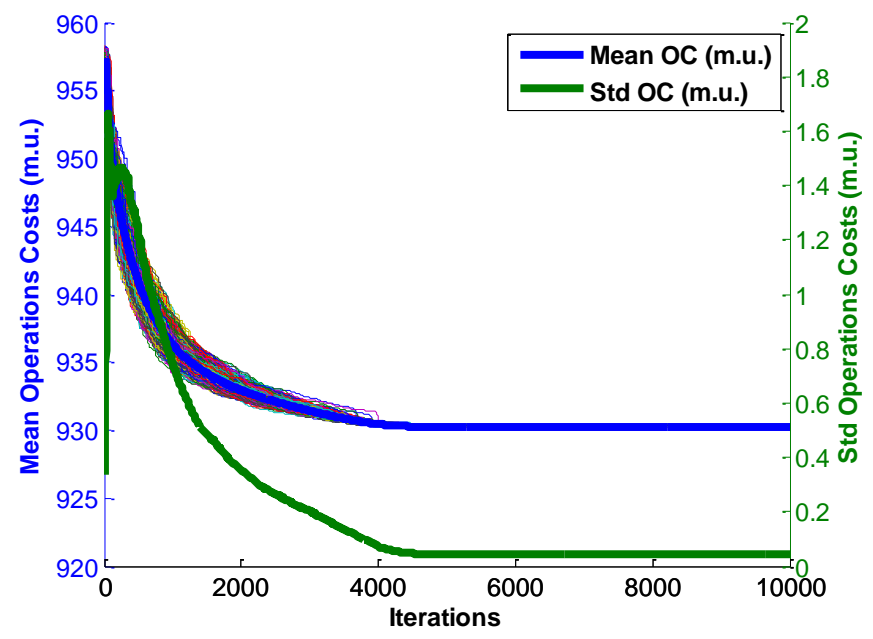

Figure 4. QPSO mean and standard deviation fitness evolution.
Fig. 4 shows evolution of mean (blue line) and standard deviation (green line) fitness in this scenario, respective to the value of operations costs. The evolution of the values of operation costs obtained in the 1000 random runs are also identified in Fig. 4 whose evolution is represented by the variety of colors less prominent.

For the same scenario, Table IV presents the results obtained by QPSO for the mean, standard deviation (std) and algorithm execution time.

TABLE IV. QPSO CHARACTERISTICS OF OBTAINED RESULTS.

\begin{tabular}{c|c|c|c|}
\hline \hline \multirow{2}{*}{ QPSO } & $\begin{array}{c}\text { Mean Operation } \\
\text { Costs }(m . u .)\end{array}$ & $\begin{array}{c}\text { Std Operation Costs } \\
(\text { m.u. })\end{array}$ & $\begin{array}{c}\text { Execution } \\
\text { Time }(s)\end{array}$ \\
\cline { 2 - 5 } & 930.26 & 0.04 & 32.50 \\
\hline \hline
\end{tabular}

In Fig. 5 are presented for each one of scenarios, the schedule of resources of each technology, for the purpose of meet the consumption required.

As can be seen in Fig. 5, the resources of DG, in all scenarios, are used to the fullest, depending on availability. Moreover, the use of the DR resource are essential to allow proper operation of the electric power system in question. The value represented by "DR programs" in Fig. 5 results from the sum of the reduced consumption by all DR programs.

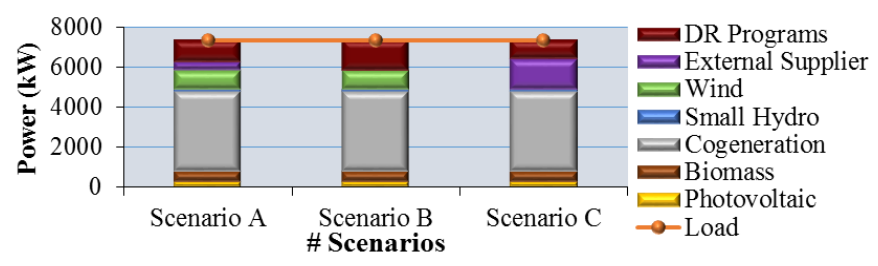

Figure 5. Scheduling each resource type for each scenario.

Fig. 6 shows for each scenario the contribution of each consumer for each type of DR program. As mentioned in Section IV, the DR programs are applied simultaneously and each consumer can participate in two distinct programs.

For each of the three scenarios presented, the value of reduced consumption is different. In Scenario A, concerns the price of energy from the external supplier is $30 \%$ more expensive than the initial, it is verified that the type of consumer that more reduces their consumption is the I consumer, through DR quadratic and steps programs. Also, the SC kind of consumers contribute with a significant percentage of the reduced consumption.

In Scenario B, the VPP cannot schedule energy from external suppliers. Once again, it is DR quadratic program that more contributes to the reduction of consumption, through the MC, LC and I consumers.

Lastly, the Scenario C corresponds to the scenario in which there is wind unavailability. The results obtained in this scenario are similar to the Scenario A, i.e. the Industrial and Small commerce consumers reduces their consumption using quadratic, linear and constant DR programs.

In all three scenarios it is possible to verify that the DR programs never reach the maximum capacity available for reduction of the consumption. 


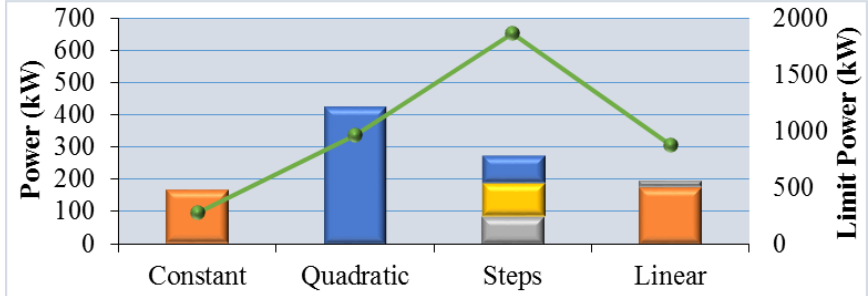

a)

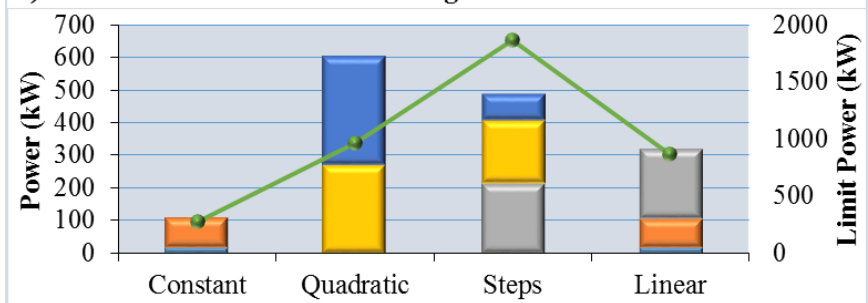

b)

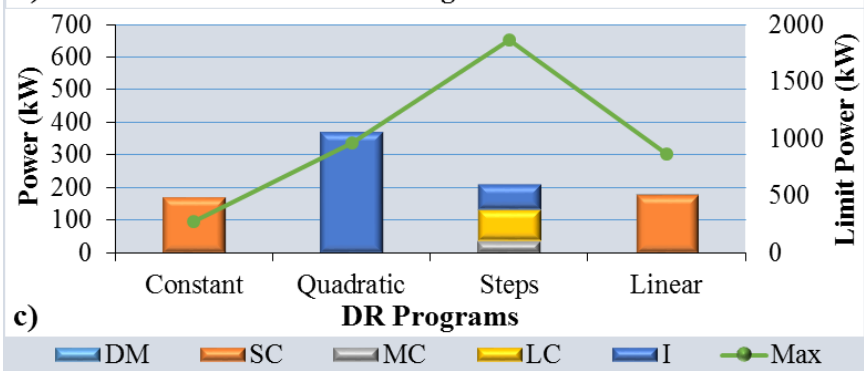

Figure 6. Consumption reduction by consumer type: a) Scenario A; b) - Scenario B; and c) - Scenario C.

\section{B. Implementation of DR Programs Individually}

In this subsection are presented the results of implementation of DR programs individually for each one of the described scenarios. This scenarios allow to analyze three critical situations operation of a network.

Fig. 7 shows the results for each of the three Scenarios (A, $\mathrm{B}$ and $\mathrm{C}$ ), previously described. For each scenario, one can see that each consumer participates in each DR program. In all three cases, DR programs that attract more consumers to reduce their consumption are the DR constant, linear and quadratic programs. These three approaches have similar results in each scenario.

With regard to consumers, it can be seen that in Scenario $\mathrm{A}$ and $\mathrm{C}$, the $\mathrm{SC}$ and $\mathrm{I}$ consumers contribute with more reduction in each $\mathrm{DR}$ program. In Scenario $\mathrm{B}$, there is a greater consumption reduction of SC, MC and $\mathrm{LC}$ as well as I consumers.

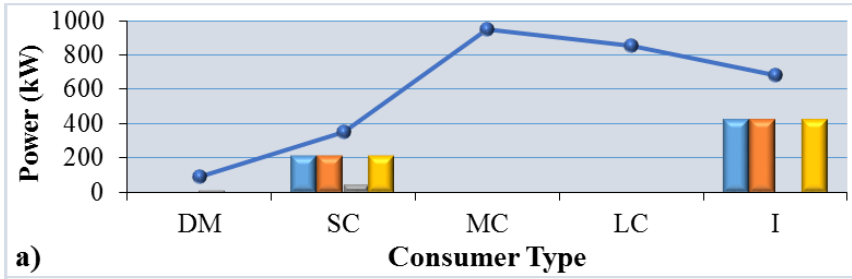

a)

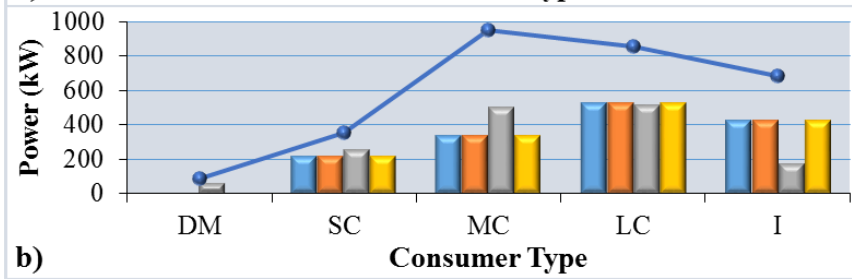

b)

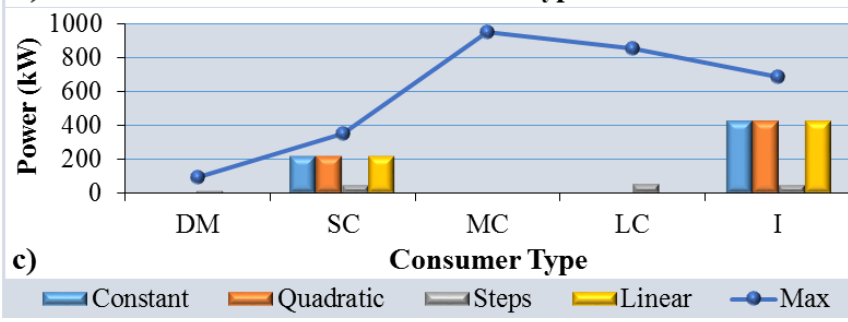

Figure 7. Consumption reduction by DR program type: a) Scenario A; b) - Scenario B; and c) - Scenario C.

In Fig. 8 is shown a particular situation of the results, where it is can see the participation of each of the DM consumers in DR steps program, when implemented individually.

The scenario $\mathrm{B}$ is the one where DM reduces more their consumption. For the remaining two scenarios (A and $\mathrm{C}$ ), the results are similar to each other, there is relatively little contribution of these consumers to consumption reduction.

\section{Comparison of the Results for Several Scenarios}

The present subsection shows one comparison of results, for the three scenarios, considering the individual and simultaneous implementation of DR programs.

Fig. 9 identifies the remuneration applied to each resource in each scenario, when DR programs are implemented individually and simultaneously. The remuneration concerning the simultaneous application of DR programs is called "All DR", while for individual implementation of DR programs is indicated by the respective program name, as shown in Fig. 9.

The total remuneration that the VPP should attain is similar for all scenarios, for both individual and simultaneous implementation of DR programs.

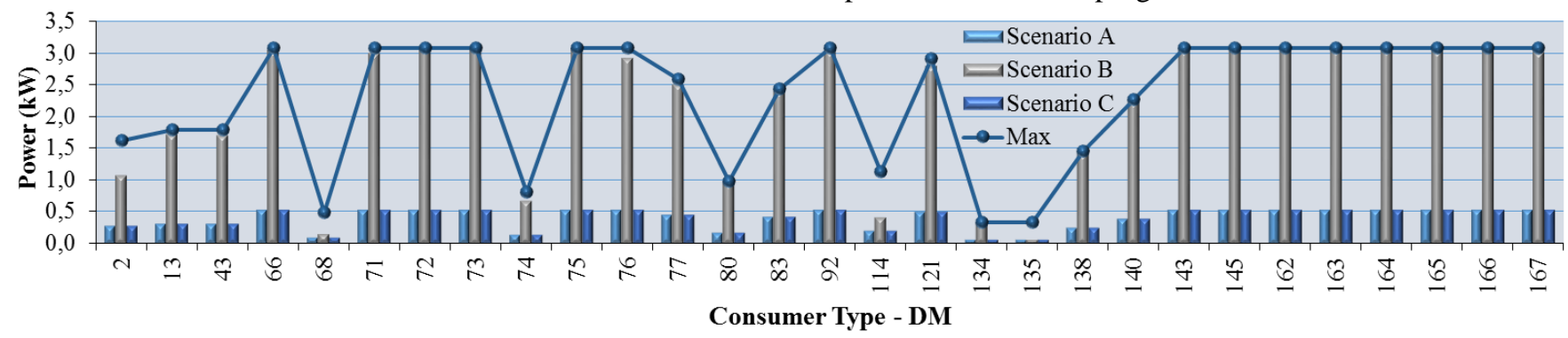

Figure 8. Consumption reduction for DM consumer type by the DR steps program type. 


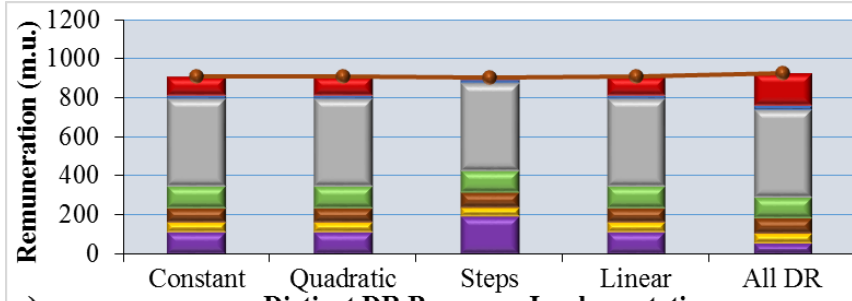

a)
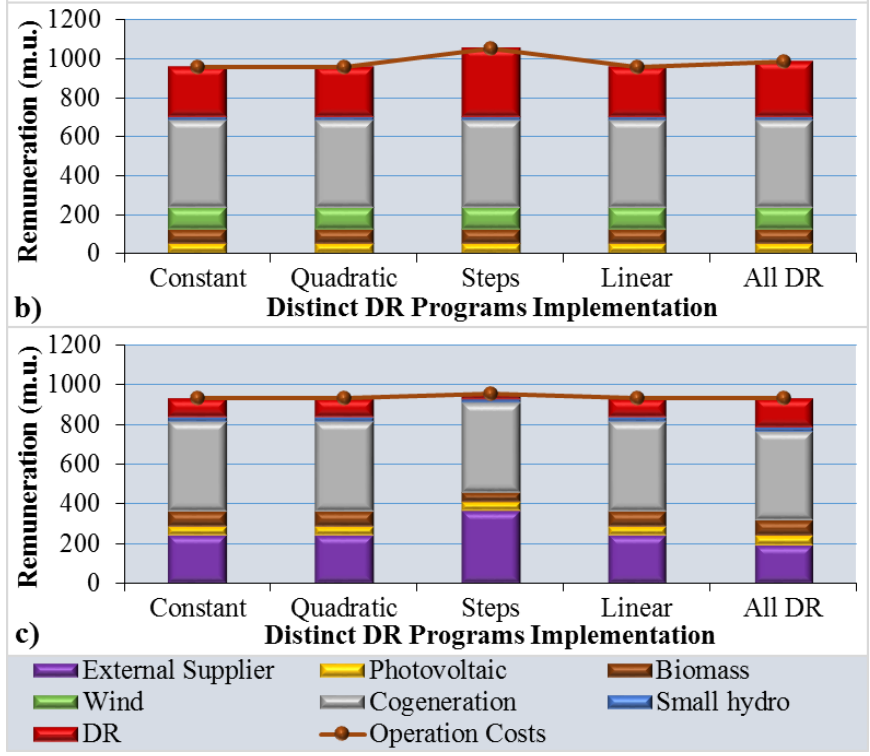

Figure 9. Remuneration by resource type for each scenario.

\section{CONCLUSIONS}

This paper aims to demonstrate several approaches of DR programs, in order to determine the remuneration and scheduling to be made to consumers. The remuneration made to consumers by participating in DR programs should be according to their characteristics, to provide a more adequate remuneration. At the same time, the aim is to show that implementing DR programs can be very useful, as it allows the VPP to handle critical situations of an electric power system. Another innovative aspect concerns the application of the Quantum Particle Swarm Optimization (QPSO) to the implemented optimization problem.

The results are obtained using the QPSO approach for the three scenarios analyzed where the VPP is able to schedule the DG, external supplier and DR programs resources to ensure high quality service. These results show that the DG resources and DR programs brings benefits to the consumers and VPP. Moreover, the performance of the algorithm is analyzed for the presented case study.

In many cases it is imperative that the implementation and studies of operation scenarios are determined in reasonable time. Thus, it is important to develop techniques able to get reasonable solutions in a timely manner, considering several complex scenarios of operation. The algorithm implemented in this paper, allows to determine satisfactory results for the optimization problem considered.

\section{REFERENCES}

[1] J. MacCormack, H. Zareipour, W.D. Rosehart, "Long-Term Market Equilibrium Model With Strategic, Competitive, and Inflexible Generation", IEEE Transactions on Power Systems, vol.27, no.4, pp.2291-2292, Nov. 2012.

[2] FERC. Staff Report, assessment of demand response and advanced metering, Dec 2014. Available: https://www.ferc.gov/legal/staffreports/2014/demand-response.pdf [accessed in May 2015].

[3] S. M. Brahma, A. A. Girgis, "Development of adaptive protection scheme for distribution systems with high penetration of distributed generation," Power Delivery, IEEE Transactions on, vol.19, no.1, pp.56,63, Jan. 2004.

[4] A.J. Gil Mena, J.A. Martín García, "An efficient approach for the siting and sizing problem of distributed generation", International Journal of Electrical Power \& Energy Systems, vol. 69, pp. 167-172, July 2015.

[5] P. Faria, T. Soares, Z. Vale, H. Morais, "Distributed generation and demand response dispatch for a virtual power player energy and reserve provision", Renewable Energy, vol. 66, pp. 686-695,June 2014.

[6] H. Morais, T. Pinto, Z. Vale, I. Praça, "Multilevel Negotiation in Smart Grids for VPP Management of Distributed Resources", IEEE Intelligent Systems magazine, Special Issue "Sustainable Energy and Distributed AI", vol. 27, no. 6, pp. 8-16, Nov. 2012.

[7] P. Faria, Z. Vale, "Demand response in electrical energy supply: An optimal real time pricing approach", Energy, Vol. 36, Issue 8, pp. 537453847, Aug. 2011.

[8] M. Nikzad, S.S.S. Farahani, "Studying emergency demand response programs based on exponential modeling in smart grids," REDEC Conference 2012, vol., no., pp.1,6, 28-29 Nov. 2012.

[9] S. S. S. FARAHANI, et al. "The Study of CPP Programs Based on Logarithmic Model in Electricity Markets". Indian Journal of Science and Technology, [S.1.], pp. 1556-1559, Nov. 2011.

[10] H.A. Aalami, M. Parsa Moghaddam, G.R. Yousefi, "Modeling and prioritizing demand response programs in power markets", Electric Power Systems Research, Vol. 80, Issue 4, pp 426-435, April 2010.

[11] P. Faria, J. Soares, Z. Vale, "Quantum-based particle swarm optimization application to studies of aggregated consumption shifting and generation scheduling in smart grids," 2014 IEEE Symposium on Computational Intelligence Applications in Smart Grid (CIASG), pp.1-8, 9-12 Dec. 2014.

[12] S.Y. Lim, M. Montakhad, H. Nouri, "Economic dispatch of power system using particle swarm optimization with constriction factor", International Journal of Innovations in Energy Systems and Power, vol.4, no.2, pp. 29-34, 2009.

[13] X. Yuan, H. Nie, A. Su, L. Wang, Y. Yuan, "An improved binary particle swarm optimization for unit commitment problem", Expert Systems with Applications, vol. 36, no. 4, pp. 8049-8055, May 2009.

[14] T.F. Araújo, W. Uturbey, "Performance assessment of PSO, DE and hybrid PSO-DE algorithms when applied to the dispatch of generation and demand", International Journal of Electrical Power \& Energy Systems, vol. 47, pp. 205-217, May 2013.

[15] Jun Sun, Choi-Hong Lai, Xiao-Jun Wu, "Particle Swarm Optimisation: Classical and Quantum Perspectives", Chapman \& Hall/CRC Numerical Analysis and Scientific Computing Series, ISBN 1439835764, 9781439835760,419 pages, 2011.

[16] Jun Sun, Wei Fang, Vasile Palade, Xiaojun Wu, Wenbo Xu, Quantumbehaved particle swarm optimization with Gaussian distributed local attractor point, Applied Mathematics and Computation, Volume 218, Issue 7, pp 3763-3775, 1 December 2011.

[17] I. J. Ramirez-Rosado and J. L. Bernal-Agustin, "Genetic algorithms applied to the design of large power distribution systems,"Power Systems, IEEE Transactions on, vol. 13, pp. 696-703, 1998. 